\title{
RANDOMIZED CONTROLLED STUDY OF SURGERY FOR EPILEPSY
}

Eighty patients with temporal lobe epilepsy were assigned at random to surgery (40 patients) or antiepileptic drug (AED) treatment only for one year (40 patients), and results were assessed by epileptologists without knowledge of treatment regimen, at the University of Western Ontario, London Health Sciences Centre, London, Ontario, Canada. Eligible patients were at least 16 years old (mean age at seizure onset $14.3+/-11.7$ years; at randomization, $35.5+/-9.4)$. In the surgical group during the year before randomization, the average monthly frequency of seizures was 5.1, number of AEDs was 6, percent with status epilepticus was 15; mesial temporal sclerosis was diagnosed by MRI in $70 \%$, and low-grade tumor, cortical dysplasia, or vascular malformation in $15 \%$. The medical group showed similar characteristics. Operation was left-sided more than right in the proportion of $2: 1$.

At one year after surgery or AED therapy only, $58 \%$ of the surgical group were free of seizures that impair awareness, whereas $8 \%$ of the medical only group were seizure-free $(\mathrm{P}<0.001)$. Post-surgical patients had continued treatment with AEDs, $22 \%$ requiring a change or increase in dose. Adverse effects of surgery in 4 patients included a small thalamic infarct, wound infection, and decline in verbal memory; there were no deaths. Visual-field defects occurred in 22 (55\%) of the surgical group. Depression occurred in $18 \%$ and $20 \%$ of the surgical and medical groups, respectively. One medical patient died (sudden unexplained). Quality of life was better in the surgical compared to the medical group, but it improved over time in both groups. The proportion of patients employed or attending school at one year was not significantly different in surgical and medical groups (56.4\% vs 38.5\%; $\mathrm{P}=0.11$ ). (Wiebe $\mathrm{S}$, Blume WT, Girvin JP et al, and Surgery for Temporal Lobe Epilepsy Study Group. N Engl I Med August 2, 2001;345:311-318). (Reprints: Dr Samuel Wiebe, London Health Sciences Centre, University Campus, 339 Windermere Rd, London, ON N6A 5A5, Canada).

COMMENT. In this series of adult patients with temporal-lobe epilepsy, the authors consider surgery to provide superior results to prolonged antiepileptic drugs alone. Although these results are promising and impressive at a 1 year follow-up, surgery has not provided the elusive "cure" for epilepsy. It bears emphasis that all surgical patients continued their anticonvulsant therapy following surgery, and that the percentage gainfully employed or at school had not changed significantly (45\% vs $56 \%$ ) and was not significantly different from the medical group (39\%). The optimal age for surgery was not addressed, a factor considered important in pediatric epilepsy centers and also emphasized in an editorial (Engel J Jr. N Engl I Med 2001;345:365-366). Early intervention in selected pediatric epilepsy cases may prevent the deterioration in intellect and social functioning that frequently attends refractory seizures requiring increasing doses of potentially toxic AEDs. In the following article, it is interesting that poor seizure control was not correlated with an increased risk of psychopathology and psychiatric symptoms.

\section{PSYCHOPATHOLOGY AND EPILEPSY}

The psychopathology of childhood epilepsy was examined by comparing symptoms in 48 children with complex partial seizures (CPS), 39 with primary generalized epilepsy with absence (PGE), and 59 nonepileptic children, aged 5 to 16 years. referred by pediatric neurologists to the UCLA Department of Psychiatry and Neuropsychiatric Institute, Los Angeles, CA. Responses on a Child Behavior Checklist (CBCL) completed by parents and the Schedule for Affective Disorders and Schizophrenia (K-SADS) administered to parent and child were compared. The 
CBLC (Achenbach, 1991) cutoff point for clinically significant pathology was 65 of a possible 113 behavioral problem items (eg Externalizing, Internalizing; aggression, depression, hyperactivity). K-SADS diagnoses were grouped as Affective/anxiety, Disruptive, Comorbid, and symptomatic (delusions, hallucinations, social withdrawal, suicidal, irritability).

Psychopathology was identified by the CBLC and K-SADS in $26 \%$ and 51\%, respectively, of the CPS and PGE patients. Compared to the nonepileptic group, children with epilepsy had significantly higher mean CBCL scores, and more diagnoses and symptoms of psychopathology. The scores and rates of pathology did not differ in the CPS and PGE groups. IQ scores were correlated with mean CBCL scores and K-SADS diagnosis in both CPS and GPE groups, and the negative association was statistically significant in CPS patients for mean total and Externalizing CBCL scores and for irritability on the K-SADS. Poor seizure control was significantly correlated with mean total and Internalizing CBCL scores but not with K-SADS diagnoses or symptoms. The K-SADS is a more sensitive test of psychopathology than the CBCL in children with CPS and PGE. (Ott D, Caplan R, Guthrie D et al. Measures of psychopathology in children with complex partial seizures and primary generalized epilepsy with absence. I Am Acad Child Adolesc Psychiatry August 2001;40:907-914). (Reprints: Dr Derek Ott, Neuropsychiatric Institute, Room 58-242b, 760 Westwood Plaza, Los Angeles, CA 90024).

COMMENT. Children with either complex partial or primary generalized epilepsies are at increased risk of having behavioral and affective disorders. Poor seizure control may not be associated with an increased risk of psychiatric diagnoses and symptoms, but the high rate of refractory epilepsy and effect of antiepileptic drugs on behavior limit the significance of this finding. A structured diagnostic interview (K-SADS) in addition to the child behavior checklist (CBCL) may be necessary to uncover psychopathology in children with epilepsy. Children with CPS like those with GPE have high rates of both externalizing (disruptive) and internalizing (affective/anxiety) disorders. The high rates of irritability, social withdrawal, suicidal ideation, and interictal hallucinations emphasize the need for psychological counseling and psychiatric intervention in children with epilepsy.

\section{TERATOGENICITY OF CARBAMAZEPINE}

The teratogenic risk after first trimester exposure to carbamazenine was determined from 210 pregnancies followed prospectively at the Israeli Teratogen Information Service, and Laboratory of Teratology, Hebrew University, Hadassah Medical School, and Ministry of Health, Jerusalem, Israel. Indications for treatment with carbamazepine included epilepsy $(80 \%)$, trigeminal neuralgia or psychiatric disorder (13\%), and were unspecified in $7 \%$ of women. Carbamazepine was the only AFD in $68 \%$, and the mean daily dose was $645+/-339 \mathrm{mg}$. Pregnancy outcome was compared with two controls, matched and general, unexposed to teratogenic agents. Mean maternal age was 29.6 years.

A twofold increase in the rate of major congenital anomalies occurred in the carbamazepine compared to the general control group (12/160 vs 18/560; relative risk 2.24). A similar trend occurred comparing the carbamazepine and matched control groups (12/160 vs 6/179; RR 2.37). Birth weight was reduced by approximately $250 \mathrm{~g}$ in carbamazepine-exposed compared to control infants. Elective terminations of pregnancy were more frequent in the carbamazepine group. The rate of major congenital anomalies was not significantly different in epileptic and nonepileptic or mono- and polytherapy groups. The congenital anomalies among carbamazepine-exposed infants showed no specific pattern. The 